\title{
The analysis and research of simulation test in Voltage Controlled Oscillator circuit
}

\author{
Hou Weizhou ${ }^{1, a^{*}}$ \\ ${ }^{1}$ (School of Physics and Electronics, Henan University, Kaifeng 475004, China \\ Min Sheng College, Henan University, Kaifeng 475004, China) \\ ahwz204@163.com
}

\begin{abstract}
Keywords: Voltage Controlled Oscillator circuit; NIMultisim10.1; voltage- frequency conversion; virtual simulation

Abstract. The Voltage Controlled Oscillator circuit had been analyzed in virtual test via NI Multisim 10.1 software. By changing the input DC voltage, the changing rule of the output waveform was observed, has read the cycle and calculate the frequency of the output waveform in conformity with the theoretical calculation results, it was concluded that the input DC voltage size has a linear relation with the output waveform frequency. When the input voltage was sawtooth wave, the output waveform frequency was varied with the sawtooth wave amplitude value change. It shows that the input voltage is either DC or sawtooth wave, the output waveform frequency is varied with the input voltage amplitude change, this further verified the working principle of voltage - frequency conversion circuit. When the NI Multisim10.1software is properly introduced electronic circuit experiment teaching, through the results of virtual simulation instance proves that it can improve the teaching effects of the circuit theory curriculum, and students can benefit a lot from the theory teaching and virtual experiment teaching.
\end{abstract}

\section{Introduction}

The function of voltage-frequency conversion circuit turns the input DC voltage into an output voltage which the frequency of it is proportional to the input voltage. It is also known as voltage-controlled oscillator circuit, referred to the voltage controlled oscillator circuit, called VCO. Generally outputs rectangular wave ${ }^{[1-2]}$. Imagine if any physical quantity through the sensor is converted to electrical signals, after pretreatment it turns into a suitable voltage signal. Then controlling VCO, and using its output drive counter to make it record the number of rectangular wave in a certain time interval. Next using the digital to display, and finally getting the digital measuring instrument of this physical ${ }^{[3]}$. As shown in Figure 1, it follows that the voltage-frequency conversion circuit is an analog/digital conversion circuit. Now VCO has been widely used in analog / digital signal conversion, FM, remote telemetry and other devices ${ }^{[4]}$. The form of circuit has charge-balanced and reset type, in this paper only discuss charge balance voltage-controlled oscillator circuit which constituted by the voltage comparator.

Sensor $\Rightarrow$ Preprocessing $\Rightarrow$ Voltage controlled oscillator $\Rightarrow$ Count display

Fig.1 Block diagram of digital measuring instruments

The basic principle of the voltage controlled oscillator circuit which consist of voltage comparator

The voltage controlled oscillator circuit are two type of working principle of the charge balance and working principle of reset . Commonly used VCO is working principle of the charge balance.

Charge balance VCO usually composed by integrator and voltage comparator and its block diagram of principle is shown in figure 2 (a). The $S$ is the electronic switch ${ }^{[5-6]}$, controlled by the output voltage $u_{\mathrm{O}}$. 


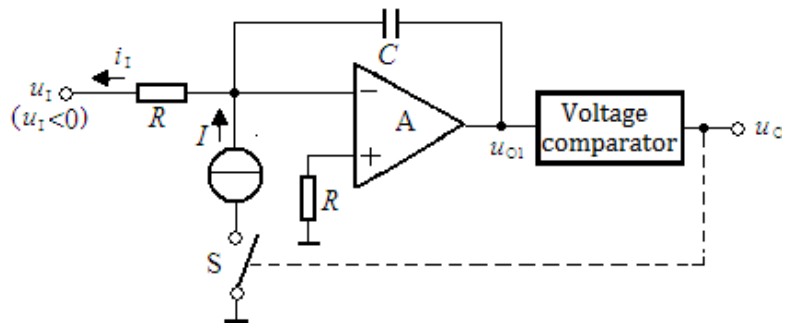

(a) Schematic

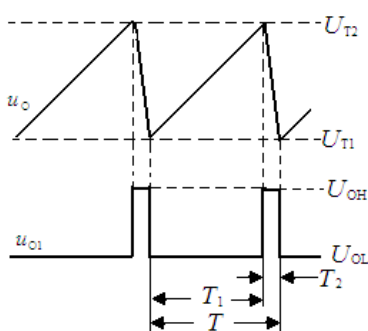

(b) Waveform Analysis

Fig. 2 Block diagram of the charge balance voltage controlled oscillator circuit.

Assuming $u_{\mathrm{I}}<0, \mathrm{I}>>\left|i_{\mathrm{I}}\right|, u_{\mathrm{O}}$ 's high level is $U_{\mathrm{OH}}$ and $u_{\mathrm{O}}$ 's low level is $U_{\mathrm{OL}}$. When $u_{\mathrm{O}}=U_{\mathrm{OH}}$, electronic switch $S$ is closed. When $u_{\mathrm{O}}=U_{\mathrm{OL}}$, the electronic switch $\mathrm{S}$ is disconnected. If the initial state of the output voltage $u_{\mathrm{O}}$ in fig. 2 is $U_{\mathrm{OL}}$, electronic switch $\mathrm{S}$ is disconnected and the integrator will integrate the input current $i_{\mathrm{I}} . i_{\mathrm{I}}=u_{\mathrm{I}} / R, u_{\mathrm{O} 1}$ is gradually increased with time, and when $u_{\mathrm{O} 1}$ increases to a certain value, $u_{\mathrm{O}}$ from $U_{\mathrm{OL}}$ jumps into $U_{\mathrm{OH}}$ to make the switch $\mathrm{S}$ closed, then the integrator doing integral computation on the difference between $I$ and $i_{\mathrm{I}}$. The difference between $I$ and $i_{\mathrm{I}}$ approximate to $I$, and $u_{\mathrm{O} 1}$ is decreased with time. Because $|I|>>\left|i_{\mathrm{I}}\right|$, the $u_{\mathrm{O} 1}$ 's decline rate is far greater than the rate of increase. Therefore, the rate of decline is far greater than the rate of increase. When $u_{\mathrm{O} 1}$ decreases to a certain value, $u_{\mathrm{O}}$ from $U_{\mathrm{OH}}$ jumps into $U_{\mathrm{OL}}$, the circuit backs to initial state. The circuit repeats this process and produce self-excited oscillation, waveform shown in Figure 2 (b) below. Because $T_{1} \gg T_{2}$, oscillation period can be considered as $T \approx T_{1}$. The larger value of $u_{\mathrm{I}}$ lets the $T_{1}$ smaller, and the frequency of oscillation higher. Thus achieving voltage-frequency converter, or achieving a voltage-controlled oscillator. Therefore, the voltage-frequency converter circuit is known as voltage-controlled oscillator circuit, same with below. Since the amount of charge which produced by the discharge(or reverse charge) of capacitor $C$ which influenced from the current source $I$ in a very short time, is equal to the amount of discharge produced by the charge of $i_{\mathrm{I}}$ in a long time. Therefore, such a circuit called a charge-balanced circuit. In a circuit which occurring sawtooth-wave, adjusting the resistance appropriately, will capable form a voltage controlled oscillator, shown in figure 3 . When $R_{\mathrm{W}} \gg>R_{3}$, the oscillation frequency $f$ is shown in equation 1 .

$$
f=R_{2} \times\left|u_{\mathrm{I}}\right| /\left(2 R_{1} R_{\mathrm{W}} U_{\mathrm{Z}} C\right)
$$

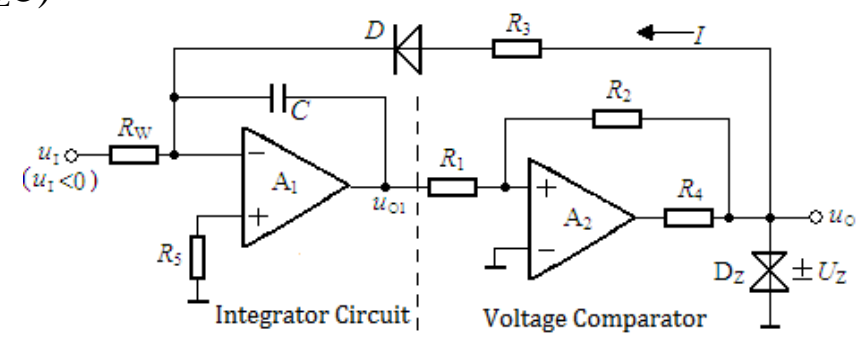

Fig.3 Sawtooth generator's evolution acts on Voltage-frequency conversion circuit.

Equation 1 illustrates the oscillation frequency $f$ is controlled by the input voltage $u_{\mathrm{I}}$, both of them appear linear relationship, concrete derivation refer to relevant books, not described in detail here.

\section{Test simulation requirements and objectives of the charge balance voltage-controlled oscillator circuit}

Build voltage controlled oscillator test circuit with charge balance type. The paper used the NI Multisim 10.1 simulation software of electronic circuit to test and analysis the voltage controlled oscillator for the type of charge balance. In accordance with the specific requirements of circuit parameters and components to build a VCO circuit, and according to the work principle of frequency-voltage conversion above described, mastered the structure and parameters of the test circuit layout simulation. Further virtual simulation of VCO circuit to observe the output waveform, read the output oscillation waveform cycle $T$ and calculated the size of corresponding frequency $f$. 
Fully grasp and understand the work principle and waveform characteristics of voltage-controlled oscillator circuit. On the basis of the sawtooth generator circuit, changing the position of potentiometer's sliding clamp. Forward integrating of integration circuit depends on the input voltage. Constituting the VCO circuit, using the work principle, related formulas and integrating circuit expression of hysteresis comparator to calculate the period $T$ of output signal waveform and frequency $f$. Observing the frequency changed laws of the output oscillating waveform of $u_{\mathrm{O}}$, which influenced by the amplitude variation of $u_{\mathrm{I}}$, when input DC voltage was sawtooth signal.

Test Simulation of the voltage controlled oscillator circuit.

(1)Respectively measuring the output waveform's period and frequency when $u_{I}$ 's amplitude equal $-6 \mathrm{~V}$ and $-3 \mathrm{~V}$, observing the changes of $u_{\mathrm{O} 1}$ 's and $u_{\mathrm{O}}$ 's waveform, then reading out the $u_{\mathrm{O}}$ 's period.

(2)Observing the features of waveform changes when $u_{\mathrm{I}}$ 's amplitude equal to the sawtooth which has the value of $-3 \mathrm{~V}$ to $-15 \mathrm{~V}$, and calculating the relationship between the amplitude variation of $u_{\mathrm{I}}$ and frequency $f$ of the output waveform.

Voltage controlled oscillator circuit's test simulation's purposes. In the paper, the test simulation of voltage controlled oscillator circuit has two purposes. First of all, mastering how the voltage-frequency converter circuit works. Secondly, researching the relationships between output waveform frequency $f$ and input voltage $u_{\mathrm{I}}$ 's amplitude of voltage controlled oscillator circuit.

\section{NI Multisim 10.1 Software's simulation test and analysis of voltage-controlled oscillator circuit}

Set up charge balanced voltage-frequency conversion of the simulation test circuit. Set up charge balanced voltage-frequency conversion of the simulation test circuit as shown in figure $4 . V_{1}$ is input DC voltage, virtual voltage source. Hysteres comparator is composed of voltage comparator $U_{2}$ and resistor $R_{1}, R_{2}$ and $R_{4}$, the high level and low level of output, in theory ,is $+5.4 \mathrm{~V}$ and $-5.4 \mathrm{~V}$ respectively. The integral circuit of sawtooth wave generator is composed of resistor $R_{3}$, virtual diode $D_{1}$, capacitor $C_{1}$. In figure 4 , integrator is composed of $R_{6}$ and capacitor $C_{1}$. Oscilloscope XSC1 observes the waveform of $u_{\mathrm{O} 1}$ and $u_{\mathrm{O}}$. Consider to the positive onset voltage of zener diode is $0.46 \sim$ $0.46 \mathrm{~V}$, the high level $U_{\mathrm{OH}}$ of output $u_{\mathrm{O}}$ is about $5.92 \mathrm{~V}$, low level $U_{\mathrm{OL}}$ is about $-5.98 \mathrm{~V}$.

When using function generator XFG1 generated sawtooth input signal, the rest of the components and parameters is like figure 4 , the test circuit as shown in figure 5.

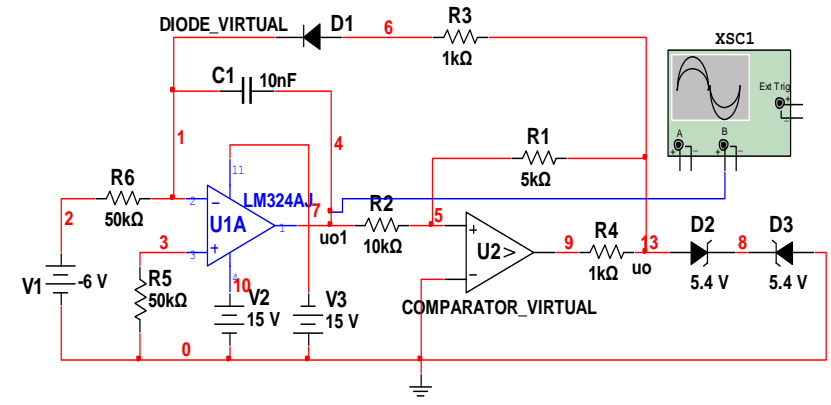

Fig. 4 VCO circuit test of $u_{\mathrm{I}}$ when the input DC voltage is $-6 \mathrm{~V}$ or $-3 \mathrm{~V}$.

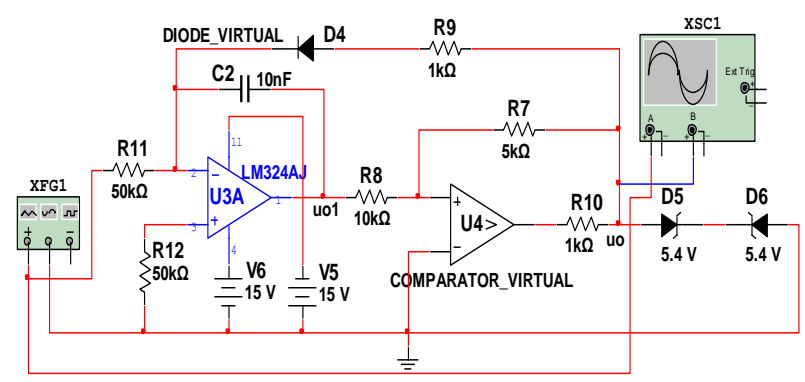

Fig. 5 VCO tests circuit when inputting a sawtooth wave signal.

\section{Test and observation of waveform changes of every circuit output}

In order to observe sawtooth wave of $u_{\mathrm{O} 1}$ and wave of $u_{\mathrm{O}}$ the period $\mathrm{T}$ and the relationship between the frequency $f$, virtual test waveform and its change rule of circuit $u_{\mathrm{O} 1}$ and $u_{\mathrm{O}}$, and illustrate VCO circuit output waveform frequency and the relationship between the input voltage range.

(1) When input DC voltage $u_{\mathrm{I}}=-6 \mathrm{~V}$, observe waveform of $u_{\mathrm{O} 1}$ as shown in figure 6 , show the waveform of $u_{\mathrm{O} 1}$ with a red line. When input DC voltage $u_{\mathrm{I}}=-3 \mathrm{~V}$, observe the waveform of $u_{\mathrm{O}}$ as shown in figure 7 , shows the waveform of $u_{\mathrm{O}}$ with a blue line. 


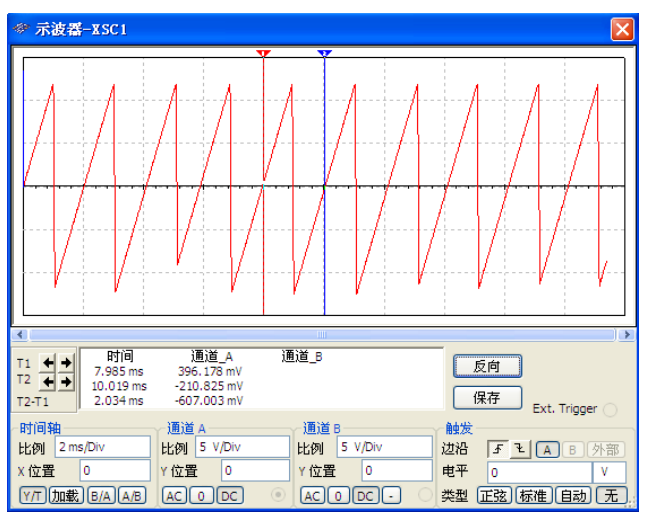

Fig. 6 Waveform of $u_{\mathrm{O} 1}$ when $u_{\mathrm{l}}=-6 \mathrm{~V}$.

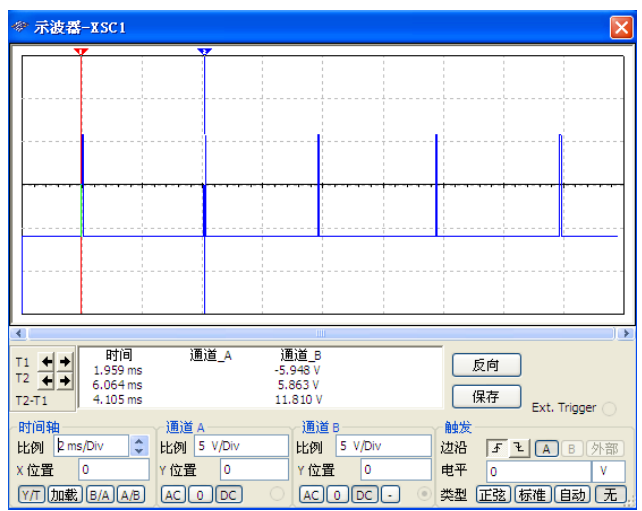

Fig. 7 Waveform of $u_{\mathrm{O}}$ when $u_{\mathrm{I}}=-3 \mathrm{~V}$.

(2) When using the function generator XFG1 to generate sawtooth wave as the signals $u_{\mathrm{I}}$, observe $u_{\mathrm{O} 1}$ and $u_{\mathrm{O}}$ waveform is shown in figure 8 , the red line shows the function generator to generate the input $u_{\mathrm{O} 1}$ sawtooth wave, the blue line shows the output $u_{\mathrm{O}}$ and density pulse waveform.

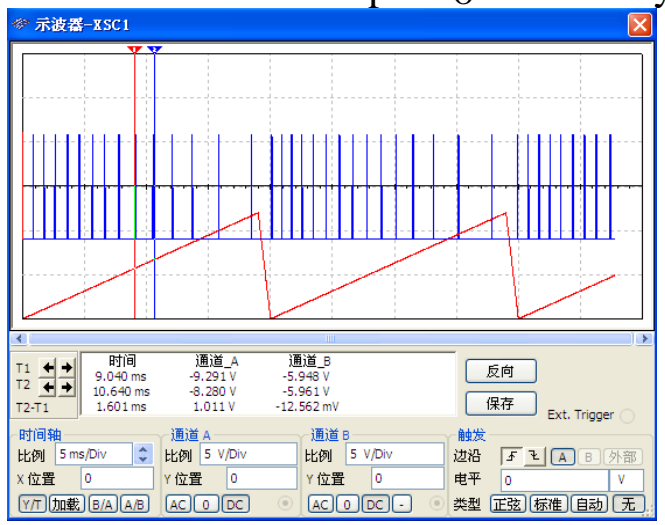

Fig. 8 Waveform of $u_{\mathrm{O} 1}$ and $u_{\mathrm{O}}$ when input is sawtooth.

\section{Test and analysis of the results of the simulation.}

(1) When using simulation test circuit in figure 4, observe the waveform of $u_{\mathrm{O} 1}$ as shown in figure 6 when $u_{\mathrm{l}}=-6 \mathrm{~V}$. The reading between two data pointer is the sawtooth wave period $\mathrm{T}, \mathrm{T}=1.601 \mathrm{~ms}$, frequency $f=491.64 \mathrm{~Hz}$, mobile data pointer readable output voltage amplitude of $u_{\mathrm{O}}$ about $\pm 5.95 \mathrm{~V}$, as shown in table 1 . Using the table 1 , calculated the frequency $f=504.2 \mathrm{~Hz}$, show that the simulation waveform cycle of reading test results agreed with theoretical calculation cycle or frequency.

(2) When the simulation test circuit $u_{\mathrm{I}}=-3 \mathrm{~V}$ in figure 4 and the waveform of $u_{\mathrm{O}}$ is as shown as that in figure 7 , the reading between two data pointer is the pulse wave period $\mathrm{T}, \mathrm{T}=4.105 \mathrm{~ms}$, frequency $f=243.61 \mathrm{~Hz}$, mobile data pointer readable output voltage $u_{\mathrm{O}}$ amplitude is $\pm 5.95 \mathrm{~V}$, as shown in table 1. Using the Table 1, calculated the frequency of $f=245.82 \mathrm{~Hz}$, show that the simulation waveform cycle of reading test results agreed with theoretical calculation cycle or frequency.

Table 1 Voltage-controlled oscillation circuit with different input voltage oscillation frequency and the output voltage amplitude

\begin{tabular}{cccc}
\hline \hline$u_{\mathrm{I}} / \mathrm{V}$ & $u_{\mathrm{O}}$ or $u_{\mathrm{O} 1}$ cycle $T / \mathrm{ms}$ & $u_{\mathrm{O}}$ or $u_{\mathrm{O} 1}$ frequency $f / \mathrm{Hz}$ & output voltage $u_{\mathrm{O}}$ amplitude $/ \mathrm{V}$ \\
\hline-6 & 2.034 & 491.64 & 5.951 \\
-3 & 4.105 & 245.82 & 5.954 \\
\hline \hline
\end{tabular}

(3) Use sawtooth wave signal generated by function generator XFG as the input $u_{\mathrm{I}}$, waveform of $u_{\mathrm{O} 1}$ and $u_{\mathrm{O}}$ is shown as figure 8 , using two readings between data pointer is the pulse wave period $\mathrm{T}$ reading, $\mathrm{T}=1.601 \mathrm{~ms}$, frequency $f=624.61 \mathrm{~Hz}$. $u_{\mathrm{O}}$ amplitude is still about $\pm 5.95 \mathrm{~V}$. Change the input signal amplitude of $u_{\mathrm{I}}$, the waveform frequency of $u_{\mathrm{O}}$ also follow changes.

(4) The figure 6 shows that when the input voltage $u_{\mathrm{I}}=-6 \mathrm{~V}$, output the frequency of voltage $u_{\mathrm{O} 1}$ and $u_{\mathrm{O}}$, waveform frequency is $491.64 \mathrm{~Hz}$. When the input voltage $u_{\mathrm{I}}=-3 \mathrm{~V}$, frequency, $f=243.61 \mathrm{~Hz}$, which frequency is about the half when $u_{\mathrm{I}}=-6 \mathrm{~V}$, this suggests that the voltage controlled oscillation circuit output waveform frequency and the input signal amplitude proportional relation, consistent with the theoretical calculation and analysis. 
(5) When the sawtooth wave produced by the function generator was used as $u_{\mathrm{I}}$, change the the amplitude of $u_{\mathrm{I}}$, frequency changes. This shows that when $u_{\mathrm{I}}=-3 \mathrm{~V} \sim-15 \mathrm{~V}$ of sawtooth wave, amplitude of $u_{\mathrm{O}}$ is $\pm 5.95 \mathrm{~V}$, changing with amplitude and frequency of $u_{\mathrm{I}}$, density and pulse wave.

\section{Conclusions}

When VCO using the input DC voltage source as input, changing the size of DC voltage amplitude of $u_{\mathrm{I}}$, the frequency of the output waveform $\mathrm{u}_{\mathrm{O} 1}$ and $u_{\mathrm{O}}$ with amplitude of $u_{\mathrm{I}}$ change size, and voltage-controlled oscillation circuit output waveform frequency $f$ is proportional to the input signal amplitude of $u_{\mathrm{I}}$. Sawtooth wave produced by the function generator and when used as the input signal is $u_{\mathrm{I}}$, change the sawtooth wave amplitude value size $-3 \mathrm{~V} \sim-15 \mathrm{~V}$, the frequency of the output waveform changes the amplitude of $u_{\mathrm{I}}$, and the output voltage $u_{\mathrm{O}}$ is about $\pm 5.95 \mathrm{~V}$, output waveform for the density and pulse wave. This verified that the result of using electronic circuit NI Multisim10.1 simulation software to virtual test voltage- frequency conversion circuit is correct. By analysing the virtual simulation of VCO circuit, using the oscilloscope observation circuit test point of each output voltage waveform changes, to find the relationship between output frequency $f$ and the amplitude of $u_{\mathrm{I}}$, further understand and master the principle of VCO, understand different analysis method and processing method of the circuit in software. Predictably introduce the simulation software to electricity class teaching, and it is the inevitable trend of teaching development. The continuous development of simulation software will continue to promote teaching method improvement, the NI Multisim 10.1 simulation experiment and traditional experiment teaching organically ${ }^{[7]}$, complement each other, give full play to the advantages of various test methods ${ }^{[8]}$, lets the student in the teaching of theory and experiment more benefit.

[Project Foundation]: Henan university the fifteenth batch of the project teaching reform funding scheme(150270084); Henan university min sheng college education teaching reform research project funding scheme(MSJG2015-12).

[About the author]: HOU Weizhou (Male; Born1973 in Yong-ji, Shanxi province) is an associate professor who engaged in application of electronic circuit in Physics and electronics institute of Henan university, Henan Kaifeng. ZIP: 475004.

\section{References}

[1] T. Shibai, H. Chengying, Analog Electronic Technology, fourth ed., Beijing, China, 2006.

[2] X. Jiakui, Electronic Circuit (Linear Portion), third ed., Beijing, China, 2004.

[3] L. Zhiqun, Electronic and Communication, Beijing, China, 2011.

[4] T. Wenxin, D. Jianguo, ect., High-Frequency Electronic Circuit, Shaanxi, China, 1996.

[5] K. Huaguang, ect., Electronic Technology Foundation (Analog Section), Beijing, China, 1999.

[7] L. Jianqing, Application of multisim software in the electric circuit experiment teaching, Journal of Zhejiang University of Technology, Vol.35, No. 5, Zhejiang, 2007, pp. 544-546.

[8] H. Weizhou, X. Bing, Simulation of Phase Locked Loop Circuit with Frequency Automatic

Tracking Based on Multisim 10. 1, Experimental Technology and Management, Vol33, No6, Beijing, 2014, pp. 86-89. 